\title{
Cardiovascular Risk Profile in Prostate Cancer Patients Treated with GnRH Agonists versus Antagonists: An Italian Real-World Analysis
}

This article was published in the following Dove Press journal: Therapeutics and Clinical Risk Management

\author{
Valentina Perronel,* \\ Luca Degli Esposti ${ }^{1, *}$ \\ Elisa Giacomini \\ Chiara Veronesi ${ }^{\prime}$ \\ Valerio Blini ${ }^{\text {I }}$ \\ Marco Oderda (iD ${ }^{2}$ \\ 'CliCon S.r.l. Health, Economics \& \\ Outcomes Research, Ravenna, Italy; \\ ${ }^{2}$ Department of Surgical Sciences - \\ Urology, Città Della Salute e Della \\ Scienza Di Torino, Molinette Hospital, \\ University of Turin, Turin, Italy
}

*These authors contributed equally to this work
Correspondence: Valentina Perrone Clicon S.r.l, Health, Economics and Outcomes Research, Ravenna, Italy Tel +3954438393

Fax +39544212699

Email valentina.perrone@clicon.it
Purpose: To evaluate and compare the incidence of cardiovascular (CV) events in a large contemporary cohort of patients diagnosed with prostate cancer $(\mathrm{PCa})$ and in treatment with GnRH agonists or GnRH antagonists.

Patients and Methods: An Italian observational retrospective cohort study based on administrative databases of three local health units and two Regions was performed. PCa patients treated with GnRH agonists or antagonist were included between January 01, 2013 and December 31, 2016. Index date (ID) was the date of first GnRH agonist/antagonist prescription during inclusion period. Follow-up was from ID to December 31, 2017. Patients were excluded if they were under abiraterone treatment or combination therapy with antiandrogens during follow-up. The incidence rate of CV events (acute myocardial infarction, ischemic heart diseases, cerebrovascular diseases, cardiac dysrhythmias, heart failure, atherosclerosis, aneurism, other CV-related conditions) was calculated among patients not switching to androgen deprivation therapy (ADT) in the overall cohort and in a sub-cohort of patients without previous CV events.

Results: In total, 9785 (mean age $76.8 \pm 8.5)$ patients were included: $9158(93.6 \%)$ were treated with a GnRH agonist and 627 (6.4\%) with a GnRH antagonist. Of them, 9627 did not switch to ADT and were considered in the analyses. The incidence of CV events was significantly higher in patients treated with GnRH agonists rather than antagonists ( 8.8 vs $6.2, \mathrm{p}=0.002$ ). Mean time to $\mathrm{CV}$ event was beyond 1 year of treatment in both groups. In the multivariable regression analysis, the risk of experiencing $\mathrm{CV}$ events was significantly lower in patients treated with GnRH antagonist rather than those treated with GnRH agonists [HR (95\% CI): $0.76(0.60-0.95), \mathrm{p}=0.018]$. These findings were confirmed in the subcohort of patients without previous $\mathrm{CV}$ events.

Conclusion: This Italian observational study shows that most patients received a GnRH agonist rather than a GnRH antagonist prescription. GnRH antagonist seems to have a better $\mathrm{CV}$ risk profile than GnRH agonist, both in patients with and without a history of CV events. Keywords: cardiovascular events, GnRH agonists, GnRH antagonist, prostate cancer, realworld setting

\section{Introduction}

Androgen deprivation therapy (ADT) is the mainstay of medical treatment of advanced prostate cancer (PCa). Although primarily indicated when evidence exists for systemic PCa, ADT is also administered in other clinical settings, including combination therapy in high-risk patients undergoing radiotherapy, findings of lymph node metastasis after radical prostatectomy, or selected locally advanced 
cancers unfit for radical treatment. ${ }^{1}$ In disagreement with contemporary guidelines, even a rise in PSA after definitive treatment is often considered as a sufficient trigger to initiate ADT. The Italian CHOICE study showed that a significant proportion of patients with $\mathrm{PCa}$ receive inappropriate ADT in terms of guideline-discordant use, exposing them to relevant side effects without survival benefits. $^{2}$

Considering the prevalence of $\mathrm{PCa}$, which is currently the most common solid tumor for men, ${ }^{3}$ a huge number of patients are exposed to ADT and its side effects. Among them, there are hot flushes, loss of libido, sexual dysfunction, fatigue, anemia, bone loss, and metabolic changes that include weight gain, insulin resistance, dyslipidemia, and lipid alterations, contributing to a significantly increased risk of diabetes and cardiovascular (CV) events. ${ }^{4,5}$ Indeed, multiple observational studies have linked ADT use to an increased risk of CV events, ${ }^{6-10}$ even if data on CV mortality remain equivocal. ${ }^{11,12}$ Based on available evidence, the Food and Drug Administration mandated the inclusion of additional safety information to GnRH agonist drug labels in 2010, whereas the European guidelines advocated special attention to the risk-to-benefit ratio of ADT in patients with a higher risk of CV complications. ${ }^{1,13}$

Among different types of ADT, GnRH antagonists might have a better $\mathrm{CV}$ risk profile compared with GnRH agonists: a pooled analysis of 2328 patients from six randomized trials of degarelix versus leuprolide found that degarelix was associated with a significantly lower risk of CV events, and this effect was particularly seen in patients with pre-existing $\mathrm{CV}$ disease. ${ }^{14}$

So far, findings are not conclusive and new evidence is highly required to address data gaps, from research on molecular mechanisms and biomarkers to clinical studies and realworld evidence generation. In light of such consideration, we structured a study with the aim to evaluate the incidence of overall CV events (acute myocardial infarction, ischemic heart diseases, cerebrovascular diseases, cardiac dysrhythmias, heart failure, atherosclerosis, aneurism, other CVrelated conditions) in a large contemporary cohort of Italian patients diagnosed with PCa treated with ADT, comparing GnRH agonists and antagonists in terms of cardiac events.

\section{Patients and Methods}

\section{Data Source}

The current real-world evidence study relied on data extracted from administrative databases from three local health units (LHU) and two Regions geographically distributed in Italy, including approximately 9,500,000 health-assisted individuals. The following databases were used: i) beneficiaries database for patient demographic characteristics; ii) pharmaceuticals database, which provides data on the Anatomical-Therapeutic-Chemical (ATC) code of the drug dispensed, drug name, number of packs and units per pack dispensed, and prescription date; iii) hospitalization database which includes all hospitalization data with primary and secondary discharge diagnosis codes classified according to the International Classification of Diseases, Ninth Revision, Clinical Modification (ICD-9-CM); iv) outpatient specialist services database, which contains all information about patients' diagnostic tests and visits. The patient code in each database allowed electronic linkage between all different databases. To guarantee the patients' privacy, an anonymous univocal numeric code was assigned to each subject included in the study, whereas study results were produced as aggregated summaries. As per Italian law, ${ }^{15}$ this study was notified to the local ethics committees of the Regions involved in the study.

\section{Study Population}

PCa patients were included if they presented at least a prescription for GnRH agonists, ATC code L02AE [buserelin (ATC code: L02AE01), leuprorelin (ATC code: L02AE02), goserelin (ATC code: L02AE03), triptorelin (ATC code: L02AE04), histrelin (ATC code: L02AE05)] or GnRH antagonist (degarelix, ATC code: L02BX02) between January 2013 and December 2016 (inclusion period). Index date (ID) was the date of first ADT prescription during inclusion period, and patients were characterized before ID (characterization period) and followed-up until December 31 ${ }^{\text {st }}, 2017$ (follow-up period). Patients were defined "ADT-naïve" if they did not have a prescription for ADT in the 2 years before ID; otherwise, they were considered "ADT-experienced". Exclusion criteria were age $\leq 20$ years, breast cancer diagnosis (identified by ICD-9-CM codes: 174, 175, 233.0, 198.81), concomitant therapy with abiraterone (ATC code: L02BX03), antiandrogens (ATC code: L0DBB), or cyproterone acetate (ATC code: G03HA01) during followup. Combination treatments were excluded in order to investigate the true effect of GnRH therapy (agonist vs antagonist) on the outcomes. To obtain a more fine-tuned investigation regarding the impact of GnRH agonist and antagonist therapy on the outcome, the same analyses were 
performed in a sub-cohort of patients without previous $\mathrm{CV}$ events.

\section{Study Variables}

Demographic and clinical characteristics were evaluated during characterization period. For each patient, the following clinical variables were considered: hypertension (at least 2 prescriptions for hypertensive drugs ATC code: $\mathrm{C} 02, \mathrm{C} 03, \mathrm{C} 07, \mathrm{C} 08, \mathrm{C} 09$ ); diabetes (at least 2 prescriptions for antidiabetics ATC code A10 or at least one hospitalization discharge diagnosis of diabetes, ICD9-CM code 250); dyslipidemia (at least 2 prescriptions for lipid-modifying agents ATC C10 and/or at least one hospitalization discharge diagnosis of disorders of lipoid metabolism, ICD-9-CM: 272 or dysmetabolic syndrome ICD-9-CM: 277.7); cardiac therapy (at least 2 prescription for drugs ATC code: C01); CV events (both primary and secondary diagnosis at discharge of: acute myocardial infarction and other forms of ischemic heart disease ICD9-CM: 410-414, cerebrovascular disease ICD-9-CM: 430-438, cardiac dysrhythmias ICD-9-CM: 427, heart failure ICD-9-CM: 428, atherosclerosis and aortic aneurysm and dissection and other aneurism ICD-9-CM: 440-442, other CV-related conditions ICD-9-CM: 401-405). Switch between GnRH agonists and antagonists was defined as the presence of a $\mathrm{GnRH}$ agonist/antagonist type of therapy during follow-up period different than that prescribed at ID. Since our aim was to investigate the effect of GnRH agonist versus antagonist therapy, patients undergoing such a switch during follow-up were excluded from analyses assessing CV events in order to obtain two "clean" treatment groups, in which the effect could be solely related to the current treatment. Mean time to switch \pm SD was estimated in months. Mean time to $\mathrm{CV}$ event $\pm \mathrm{SD}$ was expressed in years.

\section{Statistical Analysis}

Continuous variables were reported as mean and standard deviation $( \pm \mathrm{SD})$, whereas categorical variables were expressed as numbers and percentages. Incidence rate of $\mathrm{CV}$ events was calculated as the total number of CV episodes per 100 person-years in each group. Incidence rate of allcause mortality was calculated as the total number of deaths for all-cause per 100 person-years in each group. Chi-Square test was used to compare incidence of $\mathrm{CV}$ events between the two treatment groups. Cox proportional hazard model was used to estimate hazard ratios (HR) and 95\% confidence intervals (CI). The model was adjusted for potentially confounding variables: age, hypertension, diabetes, dyslipidemia, cardiac therapy and previous CV events (the latter in the overall cohort only). These variables were chosen because they can represent independent risk factors for onset of CV events. Kaplan-Meier method was applied to analyze survival to $\mathrm{CV}$ events in both treatment groups.

The risk of missing data was minimized since administrative databases capture health resources provided outside the LHU and Regions for all health-assisted patients. Given the nature of databases, no missing data were reported; therefore, no imputation techniques were used.

All analyses were performed using STATA SE, version 12.0 .

\section{Results \\ Overall Cohort}

From the original 19,858 PCa patients identified, 9785 respected the enrolment criteria, as shown in Figure 1. Among these, 9158 (93.6\%) were treated with GnRH agonists while 627 (6.4\%) were in therapy with GnRH antagonists. Of the patients included, 3497 (35.7\%) were ADT-naïve $(86.6 \%$ treated with GnRH agonists and $13.4 \%$ with GnRH antagonist) and $6288(64.3 \%)$ were ADT-experienced $(97.5 \%$ on GnRH agonists and $2.5 \%$ on $\mathrm{GnRH}$ antagonist).

Baseline characteristics of our cohort are detailed in Table 1. Mean age was 76.8 years. Most patients had hypertension $(70.1 \%)$, while only a minority had diabetes $(16.1 \%)$ or dyslipidemia (32.4\%). One thousand three hundred and thirty-four patients were under some sort of cardiac therapy (13.6\%) and 864 (8.8\%) had previously experienced a CV event. As for ADT, $93.6 \%$ of the cohort had a prescription for GnRH agonists, and 6.4\% for GnRH antagonists. Patients in the agonist group were significantly older than the antagonist group (mean 76.9 versus 74.8), while the other clinical variables considered were similarly distributed among the two groups.

Patients were followed-up for a mean time \pm SD of 3.1 \pm 1.5 years (median time [IQR] 3.3 [3.0] years). Among patients treated with GnRH agonist at ID, 61 of them switched to a GnRH antagonist during follow-up, with a mean time to switch of $20.7 \pm 13.6$ months. In the GnRH antagonist group, number of patients switching to agonist was 97 , with a mean time to switch of $19.8 \pm 13.1$ months.

The following analyses were performed considering the 9627 patients who had not switched ADT. In this population, the incidence of reported overall CV events was significantly higher in patients treated with $\mathrm{GnRH}$ agonists 


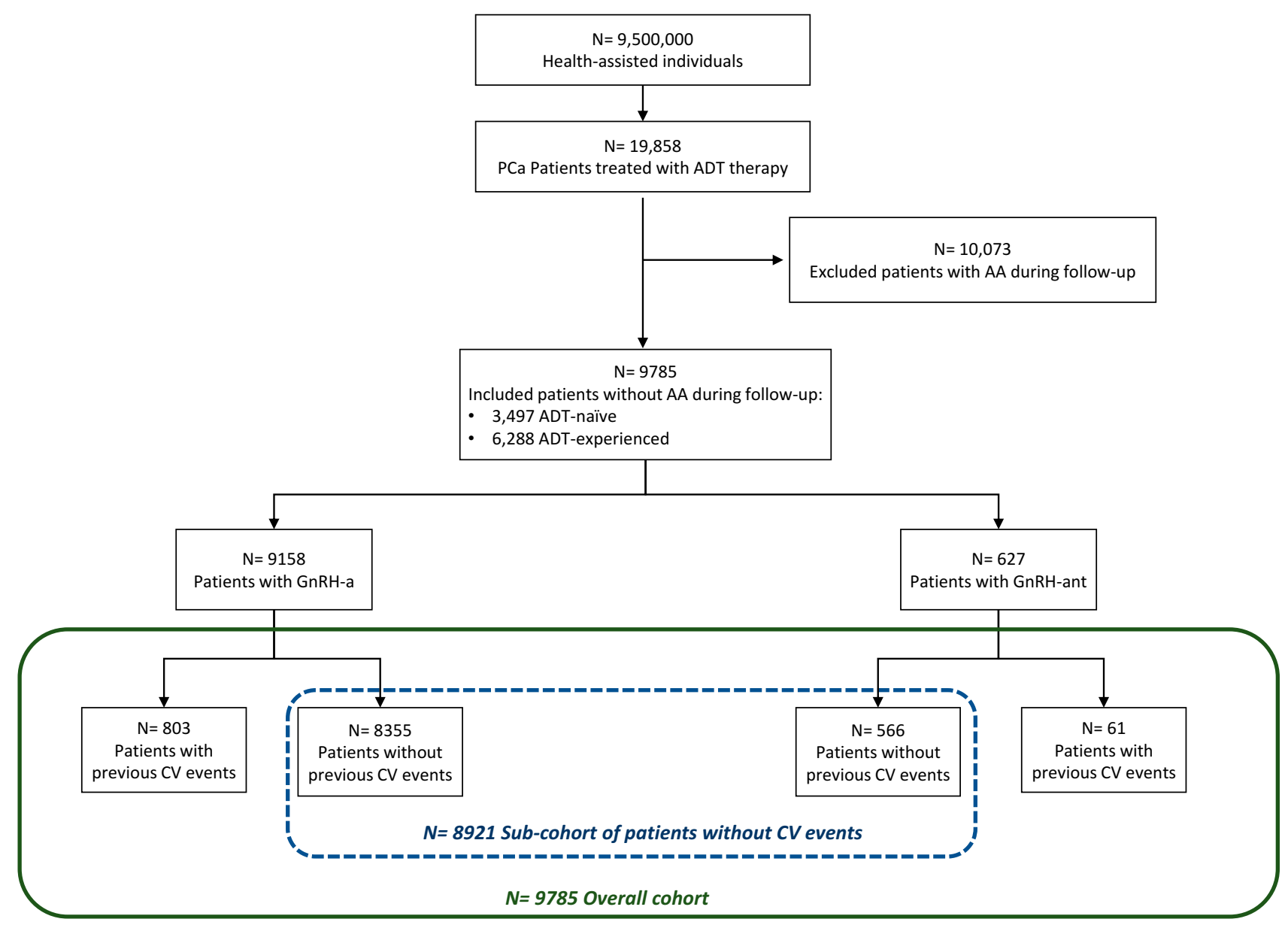

Figure I Flowchart of included patients.

Notes: Patients were defined ADT-naïve if they did not have a prescription for ADT in the 2 years before index date. Patients were defined ADT-experienced if they had at least one prescription for ADT in the 2 years before index date.

Abbreviations: AA, antiandrogens; ADT, androgen deprivation therapy; $\mathrm{CV}$, cardiovascular; GnRH-a, gonadotropin-releasing hormone agonists; GnRH-ant, gonadotropinreleasing hormone antagonist; $\mathrm{PCa}$, prostate cancer.

rather than antagonist (8.8 vs 6.2 per 100 person-years, $\mathrm{p}=0.002$ ). Mean time to $\mathrm{CV}$ event was beyond 1 year of treatment in both groups (Table 2). A similar trend was observed for all-cause mortality incidence rate, which was 9.06 and 7.83 per 100 person-years for GnRH agonist and GnRH antagonist, respectively, although the difference was

Table I Baseline Characteristics of Prostate Cancer Patients Treated with GnRH Agonists or Antagonists According to Type of Treatment

\begin{tabular}{|c|c|c|c|c|}
\hline & Study Population & GnRH Agonists & GnRH Antagonist & P Value \\
\hline Patients n (\%) & $9785(100.0)$ & 9158 (93.6) & $627(6.4)$ & \\
\hline Age (mean $\pm S D)$ & $76.8 \pm 8.5$ & $76.9 \pm 8.5$ & $74.8 \pm 8.2$ & $<0.001$ \\
\hline Age median (IQR) & $78(11)$ & $78(11)$ & $76(11)$ & \\
\hline Hypertension n (\%) & $6855(70.1)$ & $6423(70.1)$ & $432(68.9)$ & 0.513 \\
\hline Diabetes n (\%) & $1579(16.1)$ & $1483(16.2)$ & $96(15.3)$ & 0.561 \\
\hline Dyslipidemia n (\%) & $3170(32.4)$ & $2956(32.3)$ & $214(34.1)$ & 0.337 \\
\hline Cardiac therapy n (\%) & $1334(13.6)$ & $1249(13.6)$ & $85(13.6)$ & 0.954 \\
\hline Previous CV events $\mathrm{n}(\%)$ & $864(8.8)$ & $803(8.8)$ & $61(9.7)$ & 0.412 \\
\hline Follow-up period (mean years $\pm S D$ ) & $3.1 \pm 1.5$ & & & \\
\hline Follow-up period median, (IQR) & $3.3(3.0)$ & & & \\
\hline
\end{tabular}

Abbreviations: $\mathrm{CV}$, cardiovascular; $\mathrm{GnRH}$, gonadotropin-releasing hormone; IQR, interquartile; SD, standard deviation. 
Table 2 Incidence Rate of CV Events in the Overall Cohort of Prostate Cancer Patients Treated with GnRH Agonists or Antagonists

\begin{tabular}{|l|l|l|l|}
\hline & $\begin{array}{l}\text { GnRH } \\
\text { Agonist }\end{array}$ & $\begin{array}{l}\text { GnRH } \\
\text { Antagonist }\end{array}$ & P Value \\
\hline Patients $(\mathrm{n})$ & 9097 & 530 & \\
Patients with CV events ( $\mathrm{n})$ & 2206 & 76 & $<0.001$ \\
Overall number of CV events ( $\mathrm{n})$ & 5478 & 170 & \\
Time to event (mean years \pm SD) & $1.6 \pm 1.3$ & $1.2 \pm 1.1$ & \\
Time to event (median, IQR) & $1.3(1.9)$ & $1.0(1.5)$ & \\
Incidence rate/l00 person-years & 8.80 & 6.24 & 0.002 \\
\hline
\end{tabular}

Abbreviations: $\mathrm{CV}$, cardiovascular; GnRH, gonadotropin-releasing hormone; IQR, interquartile; SD, standard deviation.

not statistically significant. In the multivariable regression analysis (Cox analysis) age, hypertension, dyslipidemia, ongoing cardiac therapy, and previous $\mathrm{CV}$ events represented independent risk factors of a new CV event, while patients treated with GnRH antagonist were associated with a lower risk of experiencing $\mathrm{CV}$ events than $\mathrm{GnRH}$ agonist [HR (95\% CI): 0.76 (0.60-0.95), P value 0.018] (Table 3). The incidences of specific $\mathrm{CV}$ events as acute myocardial infarction (Supplementary Tables S1A and S3A) and stroke (Supplementary Tables S2A and S4A) were consistent with the tendency observed for overall $\mathrm{CV}$ events, although the low number of these two CV specific events did not allow to observe statistically significant differences in the two treatment groups. In the overall cohort, previous use of ADT therapy did not influence the effect of GnRH therapy: both ADT-naïve and ADT-experienced patients treated with GnRH antagonist still had a lower risk of $\mathrm{CV}$ events than the ones with agonists [HR (95\% CI): 0.789 (0.625-0.997),

Table 3 Incidence of CV Events in the Overall Cohort of Prostate Cancer Patients Treated with GnRH Agonists or Antagonists According to Characteristics at Baseline

\begin{tabular}{|l|l|l|l|l|}
\hline & HR & $\begin{array}{l}\mathbf{9 5 \%} \mathbf{~ C l} \\
\mathbf{m i n}\end{array}$ & $\begin{array}{l}\mathbf{9 5 \%} \mathbf{~ C l} \\
\mathbf{m a x}\end{array}$ & P Value \\
\hline Age & 1.05 & 1.04 & 1.06 & $<0.001$ \\
Hypertension & 1.57 & 1.40 & 1.75 & $<0.001$ \\
Diabetes & 1.06 & 0.97 & 1.16 & 0.198 \\
Dyslipidemia & 1.63 & 1.39 & 1.91 & $<0.001$ \\
Cardiac therapy & 1.58 & 1.42 & 1.75 & $<0.001$ \\
Previous CV events & 3.27 & 2.75 & 3.89 & $<0.001$ \\
\hline Treatment & & & & \\
GnRH agonists & 1.00 & & & \\
GnRH antagonists & 0.76 & 0.60 & 0.95 & 0.018 \\
\hline
\end{tabular}

Abbreviations: $\mathrm{Cl}$, confidence interval; $\mathrm{CV}$, cardiovascular; $\mathrm{GnRH}$, gonadotropinreleasing hormone HR, hazard ratio. p value 0.047] (Supplementary Table S5A). Kaplan-Meier survival curve for overall $\mathrm{CV}$ events in $\mathrm{GnRH}$ agonist and antagonist groups is shown in Figure 2A.

\section{Sub-Cohort of Patients Without Previous CV Events}

A secondary analysis focused only on a sub-cohort of patients without a history of CV events (n 8921). The baseline characteristics of this sub-cohort, which reflect the overall one, are detailed in Table 4. Among these patients, only $6.3 \%$ was treated with $\mathrm{GnRH}$ antagonist.

Considering the 8774 patients who had not switched to ADT, even in this subset of patients the incidence of reported CV events was found to be significantly lower in the antagonist group as compared to $\mathrm{GnRH}$ agonists ( $7.8 \mathrm{vs}$ 5.3 per 100 person-years, $p=0.002$ ) (Table 5). Incidence rate of all-cause mortality was 8.19 in GnRH agonists and 7.46 in $\mathrm{GnRH}$ antagonist treated patients per 100 person-years.

Among patients that did not switch to ADT therapy, the lower risk to experience a $\mathrm{CV}$ event among the $\mathrm{GnRH}$ antagonist treated patients compared to $\mathrm{GnRH}$ agonists [HR (95\% CI): $0.76(0.59-0.99)]$ (P value 0.040) was also confirmed by a multivariable regression analysis (COX analysis) evaluating age, hypertension, dyslipidemia, ongoing cardiac therapy and previous $\mathrm{CV}$ events (Table 6). This trend could be suggested also by the incidence of acute myocardial infarction (Supplementary

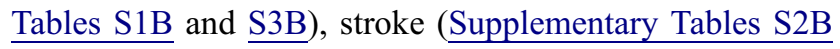
and $\underline{\mathrm{S} 4 \mathrm{~B}}$ ), and when previous use of ADT therapy was considered as a variable in the regression model (Supplementary Tables S5B) even if these analyses were not statistically significant. Kaplan-Meier survival curve for $\mathrm{CV}$ events in $\mathrm{GnRH}$ agonist and antagonist groups in this sub-cohort is shown in Figure 2B.

\section{Discussion}

ADT continues to represent the mainstay of systemic treatment for PCa but has been associated with important side effects: among them, metabolic abnormalities and CV morbidity. Several epidemiological and population-based studies have found that the use of ADT leads to an increased risk of $\mathrm{CV}$ events, that can already occur during the first year of treatment. ${ }^{7-10}$

Potential hypotheses of increased CV risk with ADT include metabolic changes caused by low testosterone, which are similar to those observed in subjects with metabolic syndrome, including hypercholesterolemia, hypertriglyceridemia, 
A

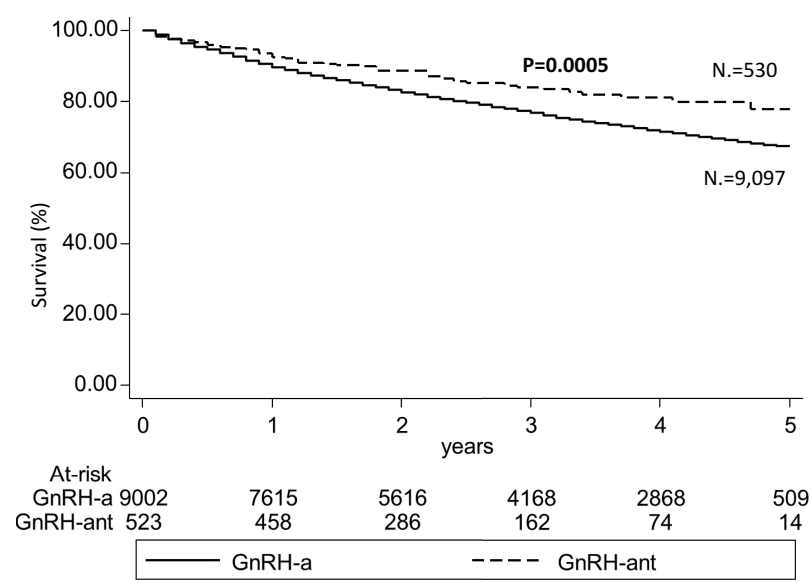

B

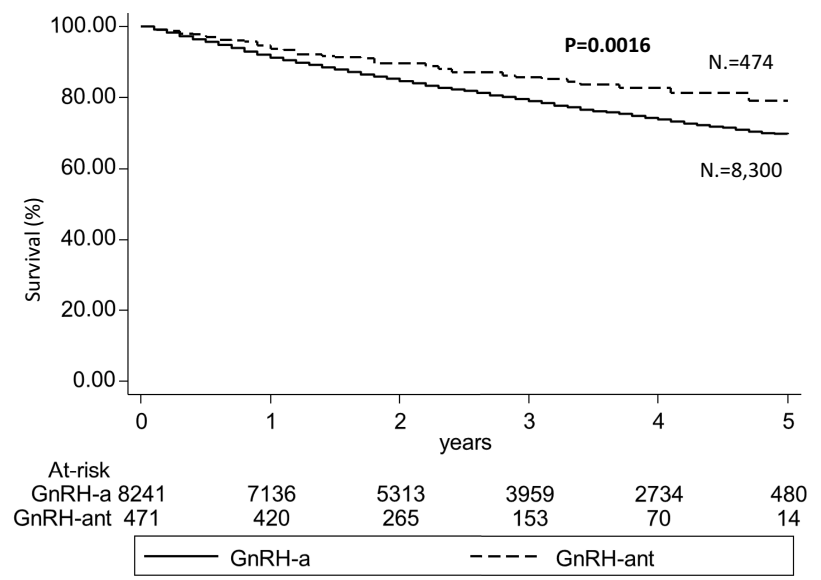

Figure 2 Kaplan-Meier survival curves of incidence of $\mathrm{CV}$ events in $\mathrm{GnRH}$ agonists and antagonist-treated patients at 5 years considering (A) overall cohort, (B) sub-cohort of patients without previous CV events.

Abbreviations: $\mathrm{CV}$, cardiovascular; $\mathrm{GnRH}-\mathrm{a}$, gonadotropin-releasing hormone agonists; $\mathrm{GnRH}$-ant, gonadotropin-releasing hormone antagonist.

and insulin resistance. ${ }^{11}$ However, long-term ADT-induced metabolic changes are unlikely to explain the differences in $\mathrm{CV}$ risk between GnRH agonists and antagonists. The difference in CV risk could, therefore, be due to the different mechanism of action: several hypotheses have been formulated to explain this outcome, mainly regarding the pathomechanisms underlying the risk of $\mathrm{CV}$ events, such as the differing effects of GnRH agonists and antagonists on FSH levels or on the GnRH receptor expressed on T lymphocytes. ${ }^{16,17}$ Preclinical data support the hypothesis that dysregulation of the FSH system plays a role in both the development and progression of prostate cancer, and could be associated to CV morbidity through the FSH receptors expressed on the luminal endothelial surface. ${ }^{18,19}$ Nevertheless, in a recent prospective Phase II randomized trial comparing $\mathrm{GnRH}$ agonist and antagonist therapies in PCa patients with a history of CV disease, ${ }^{20}$ no difference in endothelial function (primary endpoint) was observed between the two study arms. The destabilization of established vascular lesions such as atherosclerotic plaques, potentially driven by the presence of $\mathrm{GnRH}$ receptors on infiltrated T-lymphocytes, has also been proposed as a potential explanation for the diverse effect of ADT therapies on $\mathrm{CV}$ morbidity. ${ }^{21}$ Furthermore, GnRH receptors have been found in the heart, even if a direct link between cardiac GnRH receptor stimulation and GnRH agonist use has not been demonstrated. ${ }^{19}$ To date, however, these remain only interesting hypotheses to be confirmed in a clinical setting.

Despite all the observational data showing a consistent positive association between ADT and CV morbidity, post hoc analyses from randomized clinical trials (RCTs) have not confirmed this increase in CV risk. ${ }^{12,22,23}$ Methodological limitations may account for this apparent discrepancy, including selection bias, statistical approaches that did not account for competing risks, a lack of sensitivity in determining CV disease, or confounding factors. A proper

Table 4 Baseline Characteristics of Prostate Cancer Patients Treated with GnRH Agonists or Antagonists Without Previous CV Events According to Type of Treatment

\begin{tabular}{|l|l|l|l|l|}
\hline & Study Population & GnRH Agonists & GnRH Antagonist & P Value \\
\hline Patients n (\%) & $8921(100.0)$ & $8355(93.7)$ & $566(6.3)$ & \\
Age (mean \pm SD) & $76.6 \pm 8.6$ & $76.7 \pm 8.6$ & $74.8 \pm 8.3$ & $<0.001$ \\
Age median (IQR) & $77(10)$ & $78(11)$ & $76(10)$ & \\
Hypertension n (\%) & $6061(67.9)$ & $5682(68.0)$ & $379(67.0)$ & 0.606 \\
Diabetes n (\%) & $1333(14.9)$ & $1253(15.0)$ & $80(14.1)$ & 0.577 \\
Dyslipidemia n (\%) & $2718(30.5)$ & $2536(30.4)$ & $182(32.2)$ & 0.367 \\
Cardiac therapy n (\%) & $1047(11.7)$ & $981(11.7)$ & $66(11.7)$ & 0.954 \\
\hline
\end{tabular}

Abbreviations: $\mathrm{CV}$, cardiovascular; $\mathrm{GnRH}$, gonadotropin-releasing hormone; IQR, interquartile; SD, standard deviation. 
Table 5 Incidence Rate of CV Events in the Sub-Cohort of Prostate Cancer Patients Treated with GnRH Agonists or Antagonist Without Previous CV Events

\begin{tabular}{|l|l|l|l|}
\hline & $\begin{array}{l}\text { GnRH } \\
\text { Agonists }\end{array}$ & $\begin{array}{l}\text { GnRH } \\
\text { Antagonist }\end{array}$ & P Value \\
\hline Patients ( $n$ ) & 8300 & 474 & \\
Patients with CV events (n) & 1845 & 60 & $<0.001$ \\
Overall number of CV events (n) & 4245 & 140 & \\
Time to event (mean years \pm SD) & $1.7 \pm 1.3$ & $1.4 \pm 1.1$ & \\
Time to event median (IQR) & $1.4(2.0)$ & $1.1(1.5)$ & \\
Incidence rate/l00 person-years & 7.83 & 5.33 & 0.002 \\
\hline
\end{tabular}

Abbreviations: $\mathrm{CV}$, cardiovascular; GnRH, gonadotropin-releasing hormone; IQR, interquartile; SD, standard deviation.

Table 6 Incidence of CV Events in the Sub-Cohort of Prostate Cancer Patients Treated with GnRH Agonists or Antagonists Without Previous CV Events According to Characteristics at Baseline

\begin{tabular}{|l|l|l|l|l|}
\hline & HR & $\mathbf{9 5 \%} \mathbf{C l} \mathbf{m i n}$ & $\mathbf{9 5 \%} \mathbf{C l}$ max & P value \\
\hline Age & 1.06 & 1.05 & 1.06 & $<0.001$ \\
Hypertension & 1.55 & 1.38 & 1.73 & $<0.001$ \\
Diabetes & 1.09 & 0.99 & 1.21 & 0.081 \\
Dyslipidemia & 1.48 & 1.32 & 1.66 & $<0.001$ \\
Cardiac therapy & 1.59 & 1.41 & 1.79 & $<0.001$ \\
Treatment & & & & \\
GnRH agonists & 1.00 & & & \\
GnRH antagonists & 0.76 & 0.59 & 0.99 & 0.040 \\
\hline
\end{tabular}

Abbreviations: $\mathrm{Cl}$, confidence interval; $\mathrm{CV}$, cardiovascular; $\mathrm{GnRH}$, gonadotropinreleasing hormone; $\mathrm{HR}$, hazard ratio.

randomized trial evaluating the effect of ADT on CV risk and CV mortality remains to be designed, to give a definitive answer on this issue.

Among the limited evidence indicating that GnRH antagonists might have a less detrimental effect on $\mathrm{CV}$ health than GnRH agonists, ${ }^{11,14,20,24}$ a post hoc analysis gathering data from three prospective randomized trials on 2328 patients showed that GnRH antagonist halved the number of cardiac events experienced by men with pre-existing CV disease during the first year of ADT when compared to GnRH agonists, ${ }^{14}$ with a relative and absolute risk reduction of $56 \%$ and $8 \%$, respectively. Our observational data gathered from an Italian population confirm these findings, with a significantly reduced incidence of $\mathrm{CV}$ events in patients treated with GnRH antagonists versus GnRH agonists (6.2 vs 8.8 events per person-years), and a protective effect shown by multivariate analysis (HR 0.75). Interestingly, our study is the first to show that this difference is maintained also in patients without a history of $\mathrm{CV}$ disease, differently from the findings of Albertsen et al. ${ }^{14}$
Albertsen et al found that the difference of $\mathrm{CV}$ events between agonists and antagonists becomes apparent within a treatment period of 1 year, appearing more pronounced within 7 months. ${ }^{14}$ On the contrary, in our study, the time to $\mathrm{CV}$ event was more than 1 year in all study groups, suggesting that the mechanisms implied in the process are still to be fully understood.

Our results are in contrast with an observational French study based on 2010-2013 French Health Insurance data, which found no difference between agonists and antagonists in terms of risk of hospitalization for ischemic events, namely myocardial infarction or ischaemic stroke, whichever came first. ${ }^{25}$ This French study correctly took into consideration potential confounders at baseline but did not investigate the overall risk of $\mathrm{CV}$ events. More importantly, as commented by Albertsen, in that study sample size needed for statistical power was not achieved in the $\mathrm{GnRH}$ antagonist group, and therefore the hypothesis that $\mathrm{GnRH}$ antagonists lower CV complications as compared to the agonists could not be dismissed. ${ }^{26}$

Our cohort of patients reflected real clinical practice, and the results of this study must be interpreted taking into account the limitations related to the observational nature of the study based on data collected through administrative databases. Among these limitations, we highlight the lack of detailed information on comorbidities, the clinical stage of $\mathrm{PCa}$, patient lifestyle and other potential confounders that could have influenced the association between type of therapy and CV outcome. Another limitation concerned follow-up length, since patients had different follow-up times because they were enrolled at different times during inclusion period. Moreover, administrative databases do not allow to retrieve information on the cause of death; therefore, it was not possible to report if the mortality was CV- or cancer-related.

\section{Conclusion}

This Italian real-world analysis shows that the majority of patients received a GnRH agonist rather than a GnRH antagonist prescription. The methodology applied allowed the evaluation of the $\mathrm{CV}$ risk profile related to type of treatment only. The exclusion of patients with concomitant treatments for prostate cancer and of patients switching among GnRH agonists/antagonists provided indeed a clean cohort for the investigation of the effect of GnRH therapies on CV events onset. Moreover, the analyses were fine-tuned on a sub-cohort of patients without previous $\mathrm{CV}$ events. The incidence rate of $\mathrm{CV}$ events was significantly higher in the 
GnRH agonist group than in the GnRH antagonist group, both in patients without pre-existing $\mathrm{CV}$ disease and in the overall cohort. In line with this result, the regression model analysis showed a potentially better $\mathrm{CV}$ risk profile for GnRH antagonists over the agonists. The observational nature of these findings obtained in an Italian cohort through administrative database might limit their reproducibility.

\section{Acknowledgments}

Manuscript development was supported by unconditional funding from Ferring Pharmaceuticals.

\section{Disclosure}

The views expressed here are those of the authors and not necessarily those of the supporters. The agreement signed by Clicon S.r.l. and Ferring Pharmaceuticals does not create any entityship, joint venture or any similar relationship between parties. Clicon S.r.l. is an independent company. Neither Clicon S.r.l. nor any of their representatives are employees of Ferring Pharmaceuticals for any purpose. MO has worked as a consultant for Ferring and reports personal fees from Ferring, during the conduct of the study; personal fees from Janssen and Takeda, outside the submitted work. The authors report no other conflicts of interest in this work.

\section{References}

1. Cornford P, Bellmunt J, Bolla M, et al. EAU-ESTRO-SIOG guidelines on prostate cancer. part II: treatment of relapsing, metastatic, and castration-resistant prostate cancer. Eur Urol. 2017;71(4):630-642. doi:10.1016/j.eururo.2016.08.002

2. Morgia G, Russo GI, Tubaro A, et al. Prevalence of cardiovascular disease and osteoporosis during androgen deprivation therapy prescription discordant to EAU guidelines: results from a multicenter, cross-sectional analysis from the CHOsIng Treatment for Prostate canCEr (CHOICE) study. Urology. 2016;96:165-170. doi:10.1016/j. urology.2016.06.024

3. Miller KD, Nogueira L, Mariotto AB, et al. Cancer treatment and survivorship statistics, 2019. CA Cancer J Clin. 2019;69(5):363-385. doi:10.3322/caac. 21565

4. Nguyen PL, Alibhai SMH, Basaria S, et al. Adverse effects of androgen deprivation therapy and strategies to mitigate them. Eur Urol. 2015;67(5):825-836. doi:10.1016/j.eururo.2014.07.010

5. Lomax AJ, Parente P, Gilfillan C, Livingston PM, Davis ID, Pezaro C. "First, do no harm": managing the metabolic impacts of androgen deprivation in men with advanced prostate cancer. Intern Med J. 2016;46(2):141-148. doi:10.1111/imj.12731

6. Bosco C, Bosnyak Z, Malmberg A, Adolfsson J, Keating NL, Van Hemelrijck M. Quantifying observational evidence for risk of fatal and nonfatal cardiovascular disease following androgen deprivation therapy for prostate cancer: a meta-analysis. Eur Urol. 2015;68 (3):386-396. doi:10.1016/j.eururo.2014.11.039

7. Haque R, UlcickasYood M, Xu X, et al. Cardiovascular disease risk and androgen deprivation therapy in patients with localised prostate cancer: a prospective cohort study. $B r \quad J$ Cancer. 2017;117 (8):1233-1240. doi:10.1038/bjc.2017.280
8. O'Farrell S, Garmo H, Holmberg L, Adolfsson J, Stattin P, Van Hemelrijck M. Risk and timing of cardiovascular disease after androgen-deprivation therapy in men with prostate cancer. JCO. 2015;33(11):1243-1251. doi:10.1200/JCO.2014.59.1792

9. Ziehr DR, Chen M-H, Zhang D, et al. Association of androgen-deprivation therapy with excess cardiac-specific mortality in men with prostate cancer. BJU Int. 2015;116(3):358-365. doi:10.1111/bju.12905

10. Gandaglia G, Sun M, Popa I, et al. Cardiovascular mortality in patients with metastatic prostate cancer exposed to androgen deprivation therapy: a population-based study. Clin Genitourin Cancer. 2015;13(3):e123-e130. doi:10.1016/j.clgc.2014.12.003

11. Gupta D, Salmane C, Slovin S, Steingart RM. Cardiovascular complications of androgen deprivation therapy for prostate cancer. Curr Treat Options Cardiovasc Med. 2017;19(8):61. doi:10.1007/s11936-017-0563-1

12. Nguyen PL, Je Y, Schutz FAB, et al. Association of androgen deprivation therapy with cardiovascular death in patients with prostate cancer: a meta-analysis of randomized trials. JAMA. 2011;306 (21):2359-2366. doi:10.1001/jama.2011.1745

13. Research C for DE and. FDA Drug Safety Communication: Update to Ongoing Safety Review of GnRH Agonists and Notification to Manufacturers of GnRH Agonists to Add New Safety Information to Labeling Regarding Increased Risk of Diabetes and Certain Cardiovascular Diseases. FDA; June 2019. Available from:http://www.fda.gov/drugs/drugsafety-and-availability/fda-drug-safety-communication-update-ongoingsafety-review-gnrh-agonists-and-notification. Accessed July 15,2019..

14. Albertsen PC, Klotz L, Tombal B, Grady J, Olesen TK, Nilsson J. Cardiovascular morbidity associated with gonadotropin releasing hormone agonists and an antagonist. Eur Urol. 2014;65(3):565-573. doi:10.1016/j.eururo.2013.10.032

15. Agenzia Italiana del Farmaco (AIFA). Guideline for the classification and conduction of the observational studies on medicines; 2010. Available from:https://www.agenziafarmaco.gov.it/ricclin/sites/ default/files/files_wysiwyg/files/CIRCULARS/Circular\%2031st $\%$ 20May\%202010.pdf.

16. Zareba P, Duivenvoorden W, Leong DP, Pinthus JH. Androgen deprivation therapy and cardiovascular disease: what is the linking mechanism? Ther Adv Urol. 2016;8(2):118-129. doi:10.1177/1756287215617872

17. Tivesten $\AA$, Pinthus JH, Clarke N, Duivenvoorden W, Nilsson J. Cardiovascular risk with androgen deprivation therapy for prostate cancer: potential mechanisms. Urol Oncol. 2015;33(11):464-475. doi:10.1016/j.urolonc.2015.05.030

18. Radu A, Pichon C, Camparo P, et al. Expression of follicle-stimulating hormone receptor in tumor blood vessels. $N$ Engl J Med. 2010;363 (17):1621-1630. doi:10.1056/NEJMoa1001283

19. Dong F, Skinner DC, Wu TJ, Ren J. The heart: a novel gonadotrophin-releasing hormone target. $J$ Neuroendocrinol. 2011;23(5):456-463. doi:10.1111/j.1365-2826.2011.02119.x

20. Margel D, Peer A, Ber Y, et al. Cardiovascular morbidity in a randomized trial comparing $\mathrm{GnRH}$ agonist and $\mathrm{GnRH}$ antagonist among patients with advanced prostate cancer and preexisting cardiovascular disease. J Urology. 2019;202(6):1199-1208. doi:10.1097/JU.0000000000000384

21. Chen HF, Jeung EB, Stephenson M, Leung PC. Human peripheral blood mononuclear cells express gonadotropin-releasing hormone ( $\mathrm{GnRH})$, $\mathrm{GnRH}$ receptor, and interleukin-2 receptor gamma-chain messenger ribonucleic acids that are regulated by $\mathrm{GnRH}$ in vitro. $J$ Clin Endocrinol Metab. 1999;84(2):743-750. doi:10.1210/jcem.84.2.5440

22. Efstathiou JA, Bae K, Shipley WU, et al. Cardiovascular mortality after androgen deprivation therapy for locally advanced prostate cancer: RTOG 85-31. J Clin Oncol. 2009;27(1):92-99. doi:10.1200/ JCO.2007.12.3752

23. Bolla M, Van Tienhoven G, Warde P, et al. External irradiation with or without long-term androgen suppression for prostate cancer with high metastatic risk: 10-year results of an EORTC randomised study. Lancet Oncol. 2010;11(11):1066-1073. doi:10.1016/S1470-2045(10)70223-0 
24. Merseburger AS, Sedding D, Hüter K. Kardiovaskuläre Risikopatienten unter Androgenentzugstherapie. [Cardiovascular risk patients under androgen deprivation therapy: lower risk with GnRH antagonists compared to LHRH agonists?]. Urologe A. 2016;55(2):218-225. doi:10.1007/s00120-015-0013-1.German.

25. Scailteux L-M, Vincendeau S, Balusson F, et al. Androgen deprivation therapy and cardiovascular risk: no meaningful difference between $\mathrm{GnRH}$ antagonist and agonists - a nationwide population-based cohort study based on 2010-2013 French Health Insurance data. Eur J Cancer. 2017;77:99-108. doi:10.1016/j.ejca.2017.03.002
26. Albertsen PC. Re: androgen deprivation therapy and cardiovascular risk: no meaningful difference between $\mathrm{GnRH}$ antagonist and agonists. Eur J Cancer. 2017;87:203. doi:10.1016/j.ejca.2017.06.041

\section{Publish your work in this journal}

Therapeutics and Clinical Risk Management is an international, peerreviewed journal of clinical therapeutics and risk management, focusing on concise rapid reporting of clinical studies in all therapeutic areas, outcomes, safety, and programs for the effective, safe, and sustained use of medicines. This journal is indexed on PubMed Central, CAS,
EMBase, Scopus and the Elsevier Bibliographic databases. The manuscript management system is completely online and includes a very quick and fair peer-review system, which is all easy to use. Visit http://www.dovepress.com/testimonials.php to read real quotes from published authors. 